\section{Dor crônica em pacientes esquizofrênicos: prevalência e características}

\author{
Chronic pain in schizophrenic patients: \\ prevalence and characteristics
}

\author{
1 Escola de Enfermagem, \\ Universidade de São Paulo, \\ São Paulo, Brasil. \\ 2 Instituto de Psiquiatria, \\ Universidade de São Paulo, \\ São Paulo, Brasil. \\ 3 Multidisciplinary Pain \\ Center, Copenhagen \\ University Hospital, \\ Copenhagen, Denmark. \\ Correspondência \\ J. G. Almeida \\ Programa de Pós-graduação \\ em Enfermagem na Saúde \\ do Adulto, Escola de \\ Enfermagem, Universidade de \\ São Paulo. \\ Av. Dr. Enéas de Carvalho \\ Aguiar 419, São Paulo SP \\ 05403-000, Brasil. \\ joucegabriela@hotmail.com
}

\begin{abstract}
Chronic pain may be at least as prevalent in psychiatric patients as in the general population. To estimate the prevalence of chronic pain in schizophrenic patients, compare the groups with and without chronic pain, and characterize the pain, a cross-sectional study was performed on a probabilistic sample of 205 adult patients with a diagnosis of schizophrenia (mean age 37 years; $65 \%$ men; mean schooling nine years; $87 \%$ single; $65 \%$ living with parents), treated at a public hospital in the city of São Paulo, Brazil. Prevalence of pain was $36.6 \%$, and the most frequent sites were $a b$ domen (30.7\%), head, face, and mouth (24\%), and lower back (14.7\%). Twenty-four percent of patients reported feeling pain every day. Mean duration of pain was 41 months, with moderate intensity. Prevalence of chronic pain in schizophrenic patients was similar to that in the general population, and the clinical course was significant in terms of duration, intensity, and frequency.
\end{abstract}

Pain; Schizophrenia; Outpatients; Mental Health
Jouce Gabriela de Almeida 1,2 Geana Paula Kurita 1,3 Patricia Emilia Braga 1 Cibele Andrucioli de Mattos Pimenta ${ }^{1}$

\section{Introdução}

Dor crônica é freqüente na população geral e ocorre entre $20 \%$ e $40 \%$ das pessoas, considerando-se todas as idades. É um problema de saúde pública, que acarreta prejuízos pessoais e sócio-econômicos, devido a alterações nas relações interpessoais, limitação nas atividades de trabalho e uso do sistema de saúde 1. A dor crônica é aquela que persiste por mais de três meses, para fins clínicos, ou seis meses, para fins de pesquisa, de modo contínuo ou intermitente. Está relacionada a processos patológicos crônicos ou se estende além do tempo esperado para a cura de uma lesão 2,3 .

A prevalência de dor crônica entre pacientes com transtornos psiquiátricos é, possivelmente, no mínimo igual à verificada entre a população geral. No entanto, o doente mental, especialmente o esquizofrênico, parece ser pouco investigado, compreendido ou acreditado em suas queixas de dor, e diversos fatores corroboram para isso.

A falta de formação dos profissionais de saúde na área de dor crônica, a não priorização da avaliação e a falta de tratamento de queixas de dor que disso decorrem são, sem dúvida, fatores significativos. Além do mais, a experiência dolorosa pode estar relacionada ao transtorno psiquiátrico (delírio) e ser expressa de forma aberrante quanto à localização, intensidade e qualidades da dor, como descrito na esquizofrenia, o 
que confunde a compreensão e a valorização da queixa pelos familiares e profissionais.

A experiência clínica no ambulatório do Instituto de Psiquiatria do Hospital das Clínicas da Faculdade de Medicina da Universidade de São Paulo (IPq-HCFMUSP) tem mostrado que, como na população em geral, os pacientes esquizofrênicos apresentam queixas álgicas. Entretanto, não há estudo nacional sobre o assunto, e apenas quatro estudos internacionais sobre prevalência de dor crônica entre esquizofrênicos foram localizados 4,5,6,7.

Estes estudos apresentaram grande variação - de $8 \%$ a $74 \%$ - na prevalência de dor crônica em pacientes esquizofrênicos e, além disso, diferiram em seus desenhos e métodos de coleta de dados. Em três estudos, a amostra utilizada foi de conveniência 4,5,7; em dois, avaliaram-se pacientes com diferentes distúrbios psiquiátricos e não somente com esquizofrenia 4,6; em um, compararam-se pacientes esquizofrênicos com indivíduos sadios em relação à dor de cabeça 5 .

Tal diversidade entre os estudos analisados gerou o interesse de se realizar a presente investigação com os seguintes objetivos: estimar a prevalência de dor crônica em pacientes com esquizofrenia, comparar os grupos com e sem dor crônica quanto às variáveis sócio-demográficas/ doença/tratamento/uso de drogas (lícitas e ilícitas) e, por fim, caracterizar a dor.

\section{Pacientes e métodos}

\section{Desenho e população de estudo}

Realizou-se um estudo transversal com uma amostra probabilística composta por pacientes com esquizofrenia, matriculados no ambulatório do IPq-HCFMUSP, centro de referência nacional no atendimento de pacientes com transtornos psiquiátricos. A coleta de dados ocorreu no período de junho a setembro de 2008; durante esse período, 657 pacientes com esquizofrenia estavam matriculados no serviço.

Para o cálculo do tamanho da amostra foram considerados os seguintes parâmetros: prevalência de dor crônica na população geral entre $20 \%$ e $40 \% 1$ e, em pacientes psiquiátricos, $25 \%$ 8,9. Assumiu-se erro de $5 \%$ e confiabilidade de $95 \%$, perfazendo uma amostra de 201 sujeitos, aos quais foram somados $15 \%$ referentes a possíveis recusas ou perdas, obtendo-se uma amostra final de 230 doentes. A pesquisa foi aprovada pelo Comitê de Ética do HCFMUSP.

Os critérios para inclusão dos doentes na amostra probabilística foram: ter o diagnóstico médico de esquizofrenia, de acordo com a Clas- sificação Internacional de Doenças (CID-10) 10, ser regularmente matriculado no IPq-HCFMUSP, ter idade igual ou superior a 18 anos, possuir capacidade de compreensão e de verbalização para responder aos questionários, não ter história de retardo mental, não apresentar sintomas agudos no momento da entrevista (fala e comportamento desorganizados, desorientação auto e alopsíquica, agressividade e irritabilidade), concordar em participar do estudo e assinar o Termo de Consentimento Livre e Esclarecido.

Dos 230 sorteados, cinco foram excluídos por não atenderem aos critérios de diagnóstico, segundo a CID-10 10, sete por não conseguirem responder aos questionários, cinco por haverem se recusado a participar da pesquisa, um por ter falecido, sete por terem recebido alta por abandono ou por não terem sido localizados, mesmo após três tentativas. Portanto, o presente estudo refere-se a 205 pacientes que atenderam aos critérios de inclusão.

\section{Análise dos dados}

Os dados foram inseridos no programa estatístico SPSS versão 14.0 (SPSS Inc., Chicago, Estados Unidos) e analisados utilizando-se a estatística descritiva e inferencial. Calculou-se a prevalência de dor crônica entre os pacientes e seu respectivo intervalo com 95\% de confiança (IC95\%). A associação entre o desfecho (dor crônica) e as variáveis independentes foi analisada por meio do teste qui-quadrado de Pearson e, para comparar as variáveis contínuas, foi utilizado o teste $t$ de Student. Considerou-se $\alpha=5 \%$.

\section{Resultados}

Os pacientes tinham em média 37 anos (DP = $10,3)$, a maioria era do sexo masculino $(64,9 \%)$, vivia sem companheiro(a) $(87,8 \%)$, residia com os pais $(64,9 \%)$, não tinha ocupação $(63,9 \%)$ e possuía em média nove anos de escolaridade $(\mathrm{DP}=3,0)$.

Dentre os 205 pacientes esquizofrênicos avaliados, 75 apresentavam dor crônica (prevalência: 36,6\%; IC95\%: 29,9-43,6).

Na comparação entre os grupos com dor crônica e sem dor, não foi observada associação significativa com as variáveis sócio-demográficas, exceto em relação ao estado conjugal. Havia mais indivíduos sem dor crônica, que não possuíam companheiros(as) $(\mathrm{p}=0,003)$ (Tabela 1 ).

No que tange ao diagnóstico e ao tratamento da esquizofrenia, a maioria $(79,5 \%)$ tinha esquizofrenia paranóide, com um tempo médio de doença diagnosticada de 14,5 anos e tempo médio 
Tabela 1

Distribuição dos pacientes esquizofrênicos segundo características sócio-demográficas e presença de dor. São Paulo, Brasil, 2008.

\begin{tabular}{|c|c|c|c|c|c|}
\hline \multirow[t]{2}{*}{ Variáveis } & \multicolumn{2}{|c|}{ Com dor $\geq 6$ meses $(n=75)$} & \multicolumn{2}{|c|}{$\begin{array}{l}\text { Sem dor ou com dor }<6 \text { meses } \\
\qquad(n=130)\end{array}$} & \multirow[t]{2}{*}{ Valor de $p$} \\
\hline & $\mathrm{n}$ & $\%$ & $\mathrm{n}$ & $\%$ & \\
\hline Sexo & & & & & 0,086 \\
\hline Feminino & 32 & 42,7 & 40 & 30,8 & \\
\hline Masculino & 43 & 57,3 & 90 & 69,2 & \\
\hline \multicolumn{6}{|l|}{ Idade (anos) } \\
\hline Média (DP) & & & & & 0,077 * \\
\hline Mediana & & & & & \\
\hline $19-29$ & 16 & 21,3 & 39 & 30,0 & \\
\hline $30-45$ & 41 & 54,7 & 70 & 53,8 & \\
\hline $46-59$ & 12 & 16,0 & 17 & 13,1 & \\
\hline $60-69$ & 6 & 8,0 & 4 & 3,1 & \\
\hline Estado conjugal & & & & & 0,031 \\
\hline Sem companheiro & 61 & 81,3 & 119 & 91,5 & \\
\hline Com companheiro & 14 & 18,7 & 11 & 8,5 & \\
\hline Escolaridade (anos) & & & & & 0,346 * \\
\hline Média (DP) & & & & & \\
\hline Mediana & & & & & \\
\hline $0-4$ & 8 & 10,7 & 9 & 6,9 & \\
\hline $5-8$ & 18 & 24,0 & 47 & 36,2 & \\
\hline $9-11$ & 35 & 46,6 & 57 & 43,8 & \\
\hline $12-16$ & 14 & 18,7 & 17 & 13,1 & \\
\hline Reside & & & & & 0,108 \\
\hline Com os pais & 47 & 62,7 & 86 & 66,2 & \\
\hline Com o cônjuge & 14 & 8,7 & 11 & 8,5 & \\
\hline Com outros (parentes/amigos) & 8 & 10,7 & 24 & 18,5 & \\
\hline Só & 6 & 8,0 & 9 & 6,9 & \\
\hline Ocupação ** & & & & & $0,550 * \star \star$ \\
\hline Com ocupação atual & 25 & 33,3 & 49 & 37,7 & \\
\hline Superiores/Dirigentes/Gerentes & - & - & 1 & 0,8 & \\
\hline Ciências e artes & - & - & 1 & 0,8 & \\
\hline Técnicos de nível médio & 1 & 1,3 & - & - & \\
\hline Trabalhadores serviços administrativos & 1 & 1,3 & 2 & 1,5 & \\
\hline $\begin{array}{l}\text { Trabalhadores dos serviços do comércio } \\
\text { em lojas e mercados }\end{array}$ & 19 & 25,3 & 33 & 25,4 & \\
\hline Bens e serviços industriais & - & - & 3 & 2,3 & \\
\hline Reparação/Manutenção & 2 & 2,7 & 2 & 1,5 & \\
\hline Estudante & 2 & 2,7 & 7 & 5,4 & \\
\hline Sem ocupação atual & 50 & 67,7 & 81 & 62,3 & \\
\hline Nunca trabalhou & 8 & 10,7 & 13 & 10,0 & \\
\hline Auxílio-doença & 15 & 20,0 & 30 & 23,1 & \\
\hline Aposentado & 13 & 17,3 & 21 & 16,1 & \\
\hline Desempregado & 14 & 18,7 & 17 & 13,1 & \\
\hline
\end{tabular}

* Teste t de Student;

** Classificação Brasileira de Ocupações - 2002 (http://www.mtecbo.gov.br), incluindo estudantes;

*** Teste qui-quadrado: ocupação atual vs. sem ocupação atual. 
de tratamento de 13,2 anos. Verificou-se também que os pacientes tiveram, em média, duas internações psiquiátricas durante o curso da doença. Em relação a essas variáveis, também não foram observadas diferenças com significância estatística entre os grupos (Tabela 2).

A maioria $(91,7 \%)$ dos pacientes utilizou antipsicótico atípico e, destes, 13,8\% ( $\mathrm{n}=26)$ foram tratados com a associação de dois atípicos. Parcela representativa usava antidepressivos (32,2\%) e estabilizadores do humor/anticonvulsivantes (18,5\%). O clonazepam, nesses pacientes, foi utilizado como ansiolítico/benzodiazepínico (Tabela 3).

Observa-se, em todos os subgrupos medicamentosos, menor oferta de medicamentos aos doentes com dor, porém, à comparação, não houve diferença estatisticamente significativa (Tabela 3).

Verificou-se que a maioria dos pacientes não utilizava álcool (92,7\%), tabaco (80,5\%) ou drogas ilícitas $(97,6 \%)$. Os pacientes com e sem dor não diferiram quanto a essas variáveis (Tabela 4).

A dor foi mais freqüente em abdômen (30,7\%), seguido das localizações cabeça/face/boca (24\%) e região lombar/sacra/coccígea (14,7\%). Dos entrevistados, um terço referiu dor uma vez por semana, um quarto informou ter dor todos os dias e, para um quinto, a dor ocorreu uma vez por semana. O tempo médio de dor foi de 41 meses (mediana $=24$ meses). Dos 75 pacientes com dor crônica, 48 (64\%) referiram dor na última semana ou no momento da entrevista. A análise da variação da intensidade da dor, na última semana, mostrou que a mais freqüente foi moderada $(56,3 \%)$, seguida de leve $(43,7 \%)$ (Tabela 5$)$.

Ficar nervoso/ter preocupação, seguido de atividade física, foram os fatores de piora da dor mais citados. Dor espontânea ("dói sem fazer na$d a$ ”) foi referida por quase metade dos doentes. Repouso e remédios foram citados, por quase metade dos pacientes, como fatores de melhora da dor e $10 \%$ disseram que nada diminui a dor (Tabela 5).

A maioria dos pacientes relatou não ter procurado o médico $(40 / 75 ; 53,3 \%)$, não ter se queixado ao psiquiatra $(44 / 75 ; 58,7 \%)$ e não ter recebido diagnóstico $(58 / 75 ; 77,3 \%)$ e tratamento médico para dor (53/75; 70,7\%). O uso de quatro tipos de analgésicos foi citado por 11 pacientes (dipirona, butilescopolamina, diclofenaco sódico e antiespasmódico com beladona/ papaverina), e outros 11 reportaram o uso de três não analgésicos (omeprazol, ranitidina e prednisona).

Analisando-se a descrição da dor relatada pelos pacientes ("Comoéa sua dor? Com o que ela se parece?”), observou-se que os doentes souberam descrever a qualidade da dor. A quase totalidade utilizou descritores de dor semelhantes aos encontrados no Questionário de Dor McGill 11,12, e apenas um paciente descreveu sua dor de modo aberrante ("dor no olho, passa para a mente e entra na cabeça”).

Os descritores de dor utilizados foram: aguda, crônica, incomoda, anestesia, forte, fina, fraca, leve, funda, arranca, aperta, latejante, queima, cólica, pressiona, contorce, pontada, quebra, empachamento, enxaqueca, martela, cortante profunda, suportável, torcicolo, chata, pulsante, perturba, estalo, desencaixa, fincada, intermitente, desespero, inquietação, distante, machuca, mal-estar, bombando, dolorido, dolorosa, bate, agulhada, arde, tensiona, ferve, paulada, cutuca e rápida.

\section{Discussão}

Estudos sugerem que pacientes com dor crônica estão mais susceptíveis a desenvolverem problemas psiquiátricos 13,14, no entanto poucos estudos examinaram a prevalência de dor crônica nos transtornos mentais. A dor parece ser subestimada na psiquiatria, e as pesquisas são ainda menos freqüentes entre pacientes esquizofrêni$\cos 4$. Estes, em algumas situações, parecem ser menos sensíveis ao desconforto corporal, pois permanecem longos períodos em posições incômodas e contraídas, ferem-se, podem ser vítimas de queimaduras, peritonites, fraturas, infartos do miocárdio e chegam até a auto-enucleação, sem manifestar queixas de dor 15,16. Isso, embora não seja a situação mais freqüente, talvez contribua para a pequena valorização da dor desses pacientes.

No presente estudo, analisou-se a queixa álgica na última semana e no momento da entrevista, visto que os prejuízos de memória podem ocorrer em situações mais prolongadas.

Observou-se que dor crônica ocorreu em 75 dos entrevistados (36,6\%; IC95\%: 29,9-43,6), valor inferior ao descrito na Alemanha, onde a prevalência foi de aproximadamente $75 \%$ para os pacientes psiquiátricos e $74 \%$ para aqueles com transtornos psicóticos, como esquizofrenia esquizotípico, e transtornos alucinatórios 4; inferior, também, ao encontrado no estudo sobre dor de cabeça em pacientes esquizofrênicos, em que a prevalência foi de $48 \%$ entre 108 indivíduos 5. Por outro lado, esse valor é superior ao encontrado no estudo transversal realizado por Chatuverdi \& Michael 6, com 203 (14\%) pacientes psiquiátricos ambulatoriais, em que a prevalência de dor crônica foi de $8 \%$ na análise realizada com pacientes com esquizofrenia ou outras psicoses; superior, também, ao do estudo realizado 
Distribuição dos pacientes esquizofrênicos segundo diagnóstico, tempo da doença, número de internações e tempo de tratamento. São Paulo, Brasil, 2008.

\begin{tabular}{|c|c|c|c|c|c|}
\hline \multirow[t]{2}{*}{ Variáveis } & \multicolumn{2}{|c|}{ Com dor $\geq 6$ meses $(n=75)$} & \multicolumn{2}{|c|}{$\begin{array}{c}\text { Sem dor ou com dor }<6 \text { meses } \\
\qquad(n=130)\end{array}$} & \multirow[t]{2}{*}{ Valor de $\mathrm{F}$} \\
\hline & $\mathbf{n}$ & $\%$ & $\mathbf{n}$ & $\%$ & \\
\hline \multicolumn{6}{|l|}{ Diagnóstico } \\
\hline Esquizofrenia paranóide & 59 & 78,7 & 104 & 80,0 & 0,820 \\
\hline \multirow[t]{2}{*}{ Outras esquizofrenias } & 16 & 21,3 & 26 & 20,0 & \\
\hline & Média & DP & Média & DP & \\
\hline Tempo de doença & 15,43 & 7,87 & 13,90 & 7,48 & 0,169 \\
\hline Número de internações psiquiátricas & 2,09 & 3,07 & 1,80 & 2,85 & 0,491 \\
\hline Tempo de tratamento & 13,87 & 7,75 & 12,74 & 7,44 & 0,304 \\
\hline
\end{tabular}

Tabela 3

Tratamento da esquizofrenia em pacientes com e sem dor crônica. São Paulo, Brasil, 2008.

\begin{tabular}{|c|c|c|c|c|c|}
\hline \multirow[t]{2}{*}{ Medicamentos } & \multicolumn{2}{|c|}{ Com dor $\geq 6$ meses $(n=75)$} & \multicolumn{2}{|c|}{$\begin{array}{l}\text { Sem dor ou com dor }<6 \\
\text { meses }(n=130)\end{array}$} & \multirow[t]{2}{*}{ Valor de p * } \\
\hline & $n$ & $\%$ & $\mathrm{n}$ & $\%$ & \\
\hline $\begin{array}{l}\text { Antipsicóticos atípicos (olanzapina, risperidona, clozapina, } \\
\text { ziprazidona, quetiapina, amisulprida) }\end{array}$ & 67 & 35,6 & 121 & 64,3 & 0,349 \\
\hline $\begin{array}{l}\text { Antipsicóticos convencionais (haloperidol, clorpromazina, } \\
\text { trifluoperazina, levomepromazina) }\end{array}$ & 13 & 46,4 & 15 & 53,6 & 0,245 \\
\hline $\begin{array}{l}\text { Antidepressivos (fluoxetina, sertralina, venlafaxina, amitriptilina, } \\
\text { nortriptilina, paroxetina, clomipramina) }\end{array}$ & 27 & 41,0 & 39 & 59,0 & 0,376 \\
\hline $\begin{array}{l}\text { Estabilizadores de humor e anticonvulsivantes (topiramato, } \\
\text { carbolitium, carbamazepina, oxcarbazepina, valproato de sódio e } \\
\text { divalproato de sódio) }\end{array}$ & 17 & 44,7 & 21 & 55,3 & 0,248 \\
\hline $\begin{array}{l}\text { Benzodiazepínicos/Hipnóticos/Ansiolíticos (clonazepam, diazepam, } \\
\text { zolpidem, lorazepam) }\end{array}$ & 16 & 44,4 & 20 & 55,6 & 0,281 \\
\hline $\begin{array}{l}\text { Anticolinérgicos, beta-bloqueadores, anti-histamínicos, } \\
\text { antiparkisonianos (prometazina, propranolol, biperideno) }\end{array}$ & 10 & 40,0 & 15 & 60,0 & 0,705 \\
\hline
\end{tabular}

* Teste qui-quadrado.

em Londres e Edmonton, onde a prevalência de dor crônica foi de aproximadamente $18 \% 7$.

Em relação à prevalência de dor crônica na população geral adulta, nota-se igualmente grande variação nos resultados obtidos em estudos realizados em países desenvolvidos: $14 \%$ na Escócia (25 anos e mais) 17, 19\% em 15 países europeus e Israel (18 anos e mais) $18,20 \%$ na $\mathrm{Di}$ namarca (16 aos 65 anos e mais) 19 , 40\% na Suécia (18-84 anos) 20 e 46\% em Israel (25 anos e mais) 21. Em estudo realizado no Brasil com indivíduos de 22 a 69 anos, na cidade de Londrina (Paraná), observou-se que $61 \%$ tinham queixa de dor que persistia há mais de seis meses 22 . Como a dor varia com a idade e é mais freqüente nos idosos, buscou-se comparar os dados da presente pesquisa, que analisou pacientes entre 18 e 69 anos, a estudos com características semelhantes.

A prevalência de dor crônica em esquizofrênicos encontrada nesta pesquisa $(36,6 \%$; IC95\%: 29,9-43,6) está dentro dos resultados encontrados na população geral, que variaram de $20 \%$ a $61 \%$, e nos pacientes esquizofrênicos, de $8 \%$ a $74 \%$. Apesar de a morbidade ser semelhante à da população geral, a taxa de mortalidade em esquizofrênicos é maior. Talvez isso ocorra pela dificuldade de alguns pacientes em descrever adequadamente os sintomas físicos ou, princi- 
Tabela 4

Distribuição dos pacientes esquizofrênicos segundo o uso de álcool, tabaco e drogas. São Paulo, Brasil, 2008.

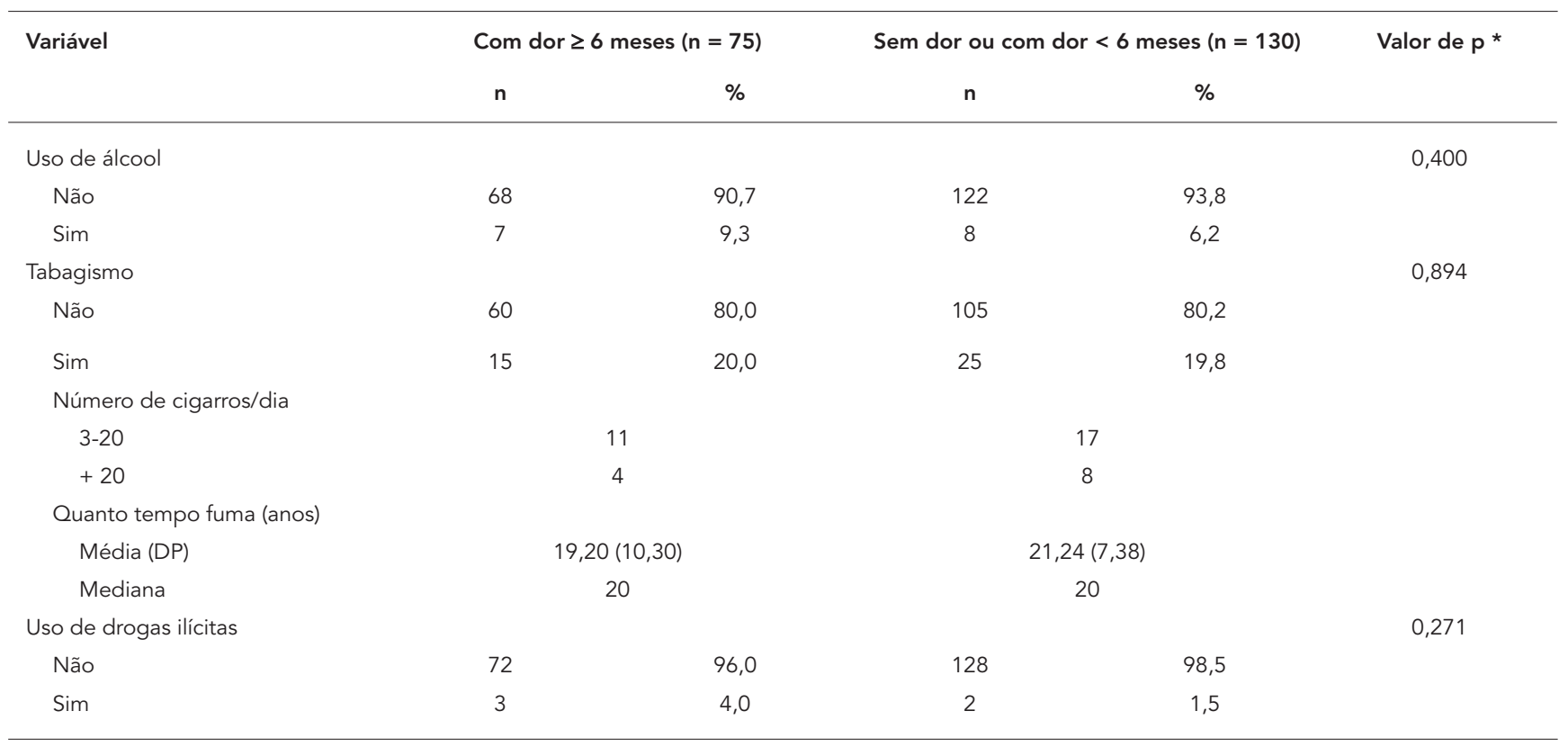

* Teste qui-quadrado.

Tabela 5

Caracterização da dor crônica por localização, freqüência, tempo de dor e intensidade. São Paulo, Brasil, 2008.

$\%$

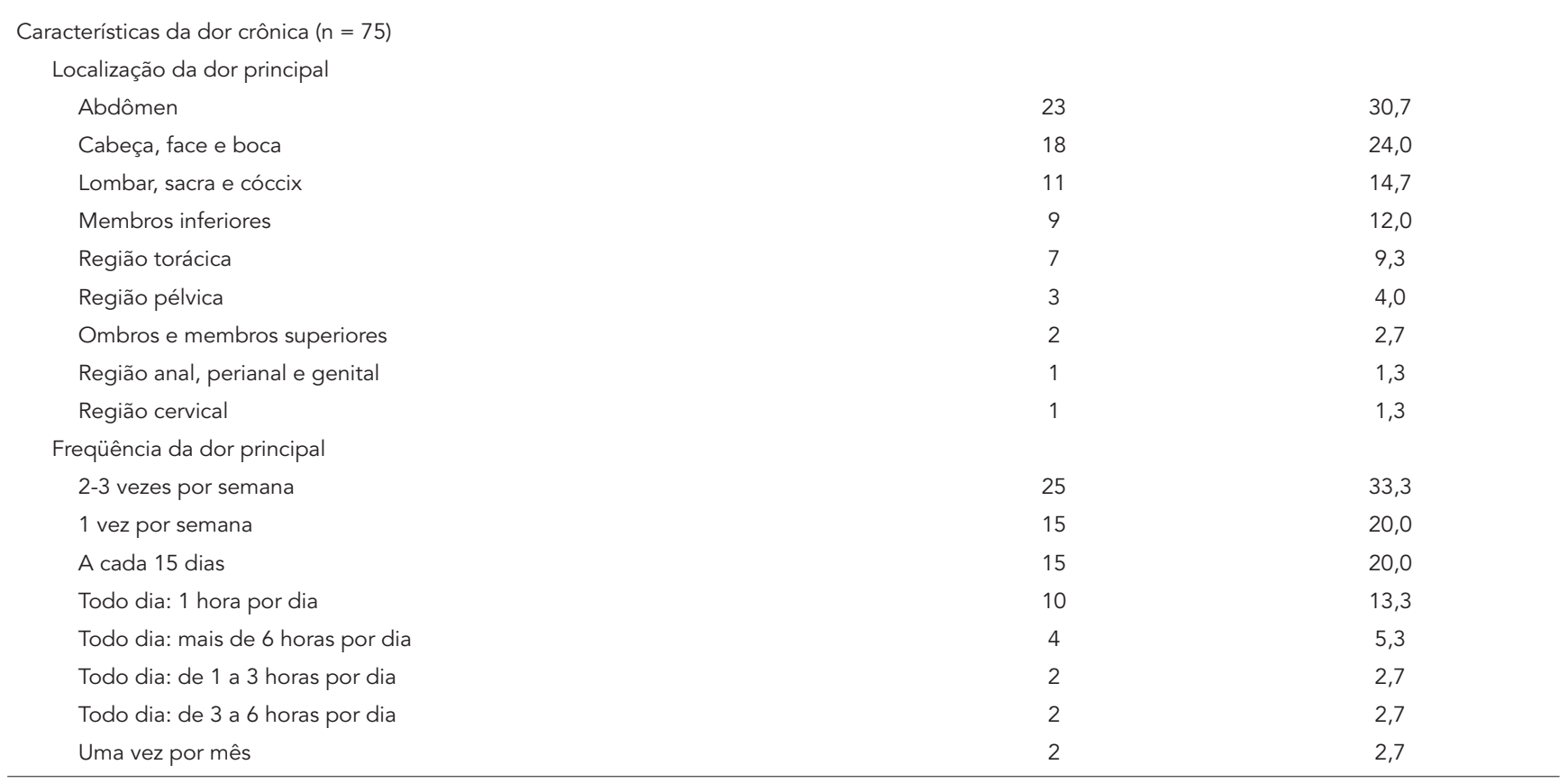

(continua) 
Tabela 5 (continuação)

n

$\%$

Tempo de dor (em meses) [Média (DP): 40,9 (42,8); Mediana: 24; Moda: 24]

6-12

13-24

$25-36$

$37-48$

49-60

$\geq 61$

Intensidade da dor na última semana e no momento da entrevista ( $\mathrm{n}=48$ ) *

Pior dor [Média (DP): 7,29 (1,68); Mediana: 8; Moda: 8]

Dor leve (1-4)

Dor moderada (5-7)

Dor intensa (8-10)

Melhor dor [Média (DP): 3,13(1,16); Mediana: 3; Moda: 3]

Dor leve (1-4)

Dor moderada (5-7)

Dor "em média" [Média (DP): 4,75 (1,19); Mediana: 5; Moda: 4]

Dor leve (1-4)

Dor moderada (5-7)

Fatores que pioram a dor

Ficar nervoso, ter preocupação

Movimentar-se

Tempo frio

Ficar sentado

Alimentar-se

Tempo quente

Dói sem fazer nada

60,0

45,1

20,0

8,0

6,7

2,7

44,0

Fatores que melhoram a dor

Repouso

Remédios

Tempo quente

Ficar calmo

Exercícios/Alongamento/Fisioterapia/Massagem

Acupuntura/Compressas

Terapia ocupacional

Nada diminui a dor

* 27 pacientes não referiram dor na última semana e nem no momento da entrevista.

palmente, pela não valorização de suas queixas pelos profissionais de saúde. No entanto, no presente estudo, a quase totalidade dos doentes descreveu adequadamente sua dor, o que se contrapõe à idéia de que a dificuldade em relatar dor seja muito freqüente.

A grande variação na prevalência de dor pode ser atribuída às diferenças nos critérios de definição de dor crônica, às características sóciodemográficas dos pacientes avaliados, às questões amostrais ou ainda a particularidades do contexto epidemiológico analisado. Além disso, o conceito de dor crônica é muito amplo (dor que dura há mais de três ou seis meses) e pode incluir pessoas com quadros muito diferentes.

Do ponto de vista metodológico, as análises de prevalência de dor crônica têm utilizado populações com características distintas, e a presença de uma ou outra variável pode influenciar na ocorrência de dor. Uma dessas variáveis é o fator etário, pois a prevalência de dor tende a aumentar conforme a idade dos indivíduos. Em estudo realizado na Suécia, a prevalência passou de $40 \%$ para $50 \%$ quando foi analisada apenas a faixa de 45 a 64 anos 20. Em estudo realizado em Pelotas (Rio Grande do Sul), encontrou-se risco 
para dor oito vezes maior ao compararem-se indivíduos de 50 a 59 anos com aqueles na faixa etária de 20 a 29 anos 23. Entre idosos (mais de 60 anos) de Londrina, observou-se a prevalência de $51 \%$ de dor crônica 24.

Outro fator relevante é o gênero do indivíduo. Embora na presente pesquisa não se tenha verificado diferença da presença de dor quanto ao gênero, observou-se tendência maior em mulheres $(\mathrm{p}=0,086)$. Dor pode ser mais freqüente em mulheres por fatores hormonais, físicos, comportamentais e psicossociais. Além das diferenças de compleição física, atividade física, mudanças hormonais e papel social da mulher, as normas sociais e culturais permitem às mulheres relatar dor mais freqüentemente, mas desencorajam os homens, ao relacionarem a tolerância à dor como medida de virilidade 1 .

Em estudo com pacientes psiquiátricos, a presença de mulheres com dor crônica foi de $56 \%$. Nos Estados Unidos, trabalho desenvolvido com a população geral identificou que $18 \%$ das mulheres e $6 \%$ dos homens apresentavam enxaqueca 25 ; em Israel, $61 \%$ das mulheres tinham dor crônica ${ }^{21}$. Em estudo realizado no Brasil com a população geral, observou-se que a dor crônica foi mais freqüente em mulheres: $69 \%$ contra $52 \%$ nos homens 22 .

Quanto à localização da dor, verificou-se, no presente estudo, que dores abdominais (31\%), seguidas das localizadas na cabeça, face ou boca $(24 \%)$, na região lombar (15\%) e em membros inferiores (12\%) foram as mais freqüentes (Tabela 5).

Ao ser comparada a estudos com pacientes esquizofrênicos de outros países, esta investigação apresentou prevalência de dor lombar semelhante à encontrada na pesquisa desenvolvida em Londres e Edmonton (18,2\%), porém muito superior ao achado do referido estudo em relação à dor abdominal (6,1\%). Já para a dor de cabeça, os achados da presente pesquisa foram semelhantes aos encontrados na Alemanha (20\%) e abaixo dos verificados no trabalho realizado em Londres e Edmonton (39,3\%) 4,7.

Comparando-se a localização da dor entre a população geral, observou-se que nossos achados equiparam-se ao estudo de Kreling et al. 22 no que se refere às queixas de dor de cabeça/face/ boca $(26,7 \%)$ e às lombares $(19,4 \%)$, que foram, respectivamente, a primeira e a segunda localização mais freqüentes. Entretanto, no presente estudo, a prevalência de dor abdominal foi muito superior à queixa de dores abdominais $(7,5 \%)$ observada no estudo de Kreling et al. 22. É possível que a alta ocorrência de dor abdominal no presente estudo esteja relacionada aos quadros de irritação gástrica por razão medicamentosa, visto que grande parte dos doentes recebia protetores gástricos.

Ainda quanto à localização da dor em estudos com a população geral, nos Estados Unidos observou-se que $15 \%$ das mulheres tinham dor pélvica crônica 26 e valor semelhante (14\%) foi encontrado na Noruega para queixas de dor no pescoço 27. Um estudo em Portugal observou que dor lombar crônica ocorreu entre $49 \%$ dos que tinham dor 28 .

À semelhança dos resultados obtidos em estudos internacionais, com a população geral, verificou-se no Brasil grande variação da prevalência de dor segundo a localização. Enquanto em Pelotas observou-se dor lombar em $4 \%$ dos entrevistados 23, em Salvador (Bahia) a prevalência foi de $15 \% 29$ e em Londrina, de $19 \% 22$. Vale destacar que, em população específica essa proporção pode ser ainda maior. Na região do vale do Aço, no Estado de Minas Gerais, 78 motoristas de ônibus urbano apresentaram 33\% de dor crônica nas costas 30 .

Observou também que, em Pelotas, aproximadamente $11 \%$ da população tinham enxaqueca, sendo essa queixa quatro vezes maior nas mulheres 31 ; já em Londrina, essa taxa foi de $27 \% 22$.

Aspecto importante da presente investigação diz respeito ao tempo, freqüência e qualidades da dor, entre outras características. Dos entrevistados, um terço (33\%) referiu dor duas a três vezes por semana, um quarto (24\%) informou ter dor todos os dias e, para um quinto, a dor ocorreu uma vez por semana, tendo 48 (64\%) referido dor na última semana ou no momento da entrevista (Tabela 5).

Na Grécia, estudo realizado com trabalhadores observou $33 \%$ para dor no momento da entrevista, $38 \%$ considerando dor há um ano, $42 \%$ há dois anos e $62 \%$ durante toda a vida ${ }^{32}$. No presente estudo, o tempo médio de dor foi 41 meses (mediana $=24$ meses) e 11\% tinham dor há pelo menos cinco anos. Em Londres e Edmonton, a prevalência de pacientes esquizofrênicos com dor há cinco anos foi de $17 \% 7$.

A duração e a freqüência da dor influem na vida do indivíduo. Dores muito prolongadas e freqüentes desgastam quem as sente e os seus familiares; exigem procura extra do serviço de saúde, de tratamentos complementares; oneram financeiramente a família e a sociedade; consomem a energia para as atividades de vida diária e, possivelmente, a disposição para as atividades de reabilitação da doença mental.

Alguns autores acreditam que pacientes esquizofrênicos estão sujeitos a ter dor de cabeça, como a população geral, e são capazes de referir sua dor quanto à duração, intensidade efreqüên- 
cia, mas, em virtude do seu comportamento e das reações subjetivas, não referem queixas 5 . Os achados da presente pesquisa confirmam essas premissas, pois a freqüência de dor observada correspondeu à da população geral, e todos os doentes descreveram sua dor com propriedade, utilizando, inclusive, descritores de dor semelhantes aos da população geral. Cerca da metade dos que tinham dor não relatou o quadro ao psiquiatra e ficou sem tratamento específico.

Dor tem qualidades sensitivas, afetivas e cognitivo-avaliativas 11,12 . Os doentes da presente pesquisa descreveram sua dor com todas essas características, empregando termos que são universais. A qualidade sensitiva da dor (em cólica, em aperto, em queimação etc.), muitas vezes, indica a etiopatogenia do quadro. Dor em aperto, em cólica, em torção, freqüentemente indica processos em vísceras ocas; dor em queimação, em calor, em choque, freqüentemente relacionase a quadros onde há neuropatias; dor descrita como funda, pesada, freqüentemente refere-se a situações com envolvimento de estruturas musculares profundas. As qualidades afetivas da dor (medo, raiva, culpa, hostilidade, tristeza) indicam o componente emocional que todo quadro doloroso tem; já o componente cognitivo-avaliativo representa a avaliação geral da experiência (incomoda, chateia, perturba).

A intensidade da dor é outro aspecto relevante. Nesta pesquisa, a análise da variação da intensidade, na última semana, mostrou que a dor mais freqüente foi a moderada $(56,3 \%)$, seguida da leve $(43,7 \%)$ (Tabela 5). Dores mais intensas tendem a ser mais incapacitantes, e a magnitude da queixa é fator importante no relato aos profissionais e familiares, na definição da terapêutica analgésica (tipo e dose) e na decisão de encaminhar, ou não, o paciente a especialistas.

A dor foi também analisada em três situações: melhor dor, pior dor e dor "em média", pois se sabe que a intensidade da dor flutua ao longo dos dias ou do dia e que conhecer essa flutuação auxilia na decisão terapêutica. Observou-se, na maior parte das vezes, que a "dor em média" foi moderada (entre 5 e 7), relatada por $56,3 \%$ daqueles com dor. Ainda, cerca de $12 \%$ dos doentes, mesmo na situação de melhor dor, referiram dor moderada e cerca de $30 \%$, mesmo na situação de pior dor, referiram dor moderada (Tabela 5). Tais dados indicam que a dor moderada foi prevalente.

A magnitude da dor observada na presente investigação assemelha-se ao que foi encontrado nos estudos com população geral, em Israel e em países europeus, em que 59\% (entre 4-7) 21 e 66\% (entre 5-7) 18 apresentaram dor moderada.
Embora o uso de psicotrópicos (alguns antidepressivos e neurolépticos) e as características da esquizofrenia sejam apontados como capazes de interferir no tratamento da dor, pelo efeito analgésico (psicotrópicos) ou pela dificuldade de se comunicar de maneira clara e objetiva 15,33, esses fatores não foram observados nesta pesquisa. Não houve diferença quanto ao uso de psicotrópicos entre doentes com e sem dor, e todos, exceto um doente, descreveram seu quadro álgico com propriedade. É possível que, nas situações de crise da doença, a interpretação e expressão de sintomas físicos estejam prejudicados, mas, nessa amostra de doentes em tratamento ambulatorial, isso não foi observado.

Outra questão pertinente refere-se aos fatores que melhoram ou pioram o estado de dor do paciente. Neste estudo verificou-se que motivos que levam à preocupação ou ao nervosismo são os que mais agravam a dor, ao passo que o descanso e a terapia medicamentosa com analgésicos aliviam a dor. Já no estudo de Israel, para aliviar a dor, $47 \%$ usavam acupuntura, $16 \%$ reflexologia, $14 \%$ shiatsu e $22 \%$ quiropraxia 21 ; na Europa, dois terços utilizavam tratamentos nãomedicamentosos, $30 \%$, massagem, $21 \%$, fisioterapia, e $13 \%$, acupuntura 18 .

Fatores emocionais são apontados como causa importante de piora ou melhora da dor em todas as populações. A percepção da dor sofre influência de fatores emocionais, cognitivos e ambientais, além dos físicos 3,34. Assim, esse relato está adequado à fisiopatologia da dor e não é exclusivo de pacientes com transtornos mentais.

O relato de que analgésicos e repouso aliviam a dor também é freqüente em outras populações. Dores músculo-esqueléticas, que são prevalentes na população, pioram com o aumento de carga (andar, levantar, abaixar, carregar objetos); por isso consegue-se a melhora com o repouso ${ }^{35}$. No estudo realizado na Europa 18, quase metade dos pacientes com dor crônica ingeriu analgésicos não-prescritos, antiinflamatórios (AINEs 55\%), paracetamol (43\%) e opióides fracos (13\%); apenas dois terços tinham prescrição médica.

No presente estudo, a dor foi freqüente (ocorreu em $36,6 \%$ ), teve duração prolongada (mediana e moda $=24$ meses), foi de intensidade moderada (56\%); $24 \%$ relataram episódios álgicos, no mínimo, diários e $20 \%$ referiram dor ao menos uma vez por semana. Isso demonstra a relevância do quadro álgico desse grupo. 


\section{Conclusão}

O estudo permitiu identificar a prevalência e as características da dor crônica em pacientes esquizofrênicos em tratamento ambulatorial, comparando-se os grupos com e sem dor crônica quanto às variáveis sócio-demográficas/doença/ tratamento/uso de drogas (lícitas e ilícitas).

Dor crônica ocorreu em mais de um terço da amostra $(36,6 \%, \mathrm{n}=75)$. A duração da queixa foi prolongada (média $=40,9, \mathrm{DP}=42,8$, mediana e moda $=24)$ e os episódios álgicos freqüentes (77,3\% dor ao menos uma vez/semana e $24 \%$ dor diária). Os locais da queixa dolorosa, excetuando o abdômen, foram semelhantes aos da população geral, para a mesma faixa etária (abdômen = $30,7 \%$, cabeça, face e boca $=24 \%$ e região lombar, sacra e cóccix $=14,7 \%)$. A intensidade da dor foi significativa. Na maior parte dos episódios, ela foi moderada (entre 5 e 7). Assim como na população geral, o humor (nervoso/ preocupado) e o aumento de carga (andar, abaixar) pioraram a dor, enquanto o repouso e remédios a aliviaram.

A prevalência e as características da dor em pacientes esquizofrênicos foram significativas $\mathrm{e}$ assemelharam-se às da população geral. A comorbidade dor crônica pode ser muito deletéria aos doentes esquizofrênicos, o que exige atenção a essa queixa. Este estudo, pioneiro em nosso meio, contribuiu de forma inédita, em âmbito nacional e, em diversos aspectos, internacional, para a construção do conhecimento sobre a prevalência e as características da dor crônica em esquizofrênicos.

\section{Resumo}

A prevalência de dor crônica entre pacientes com transtornos psiquiátricos é, possivelmente, no mínimo igual à encontrada entre a população geral. Para estimar a prevalência de dor crônica em pacientes com esquizofrenia, comparar os grupos com e sem dor crônica e caracterizar a dor foi realizado um estudo transversal, com uma amostra probabilística de 205 pacientes adultos, com diagnóstico de esquizofrenia (idade média 37 anos, $65 \%$ homens; média de escolaridade de nove anos; $87 \%$ sem companheiro(a); 65\% residentes com os pais), atendidos em hospital público do Município de São Paulo, Brasil. A prevalência de dor foi de 36,6\%; a dor foi mais presente no abdômen (30,7\%), seguida da dor de cabeça, face e boca (24\%) e região lombar, sacra e cóccix (14,7\%). Com relação à freqüiência, $24 \%$ dos entrevistados referiram ter dor todos os dias. O tempo médio de dor foi de 41 meses, com intensidade moderada. A prevalência de dor crônica em pacientes esquizofrênicos foi semelhante à encontrada para a população geral, e o quadro álgico foi significativo em termos de duração, intensidade e freqüência dos episódios dolorosos.

Dor; Esquizofrenia; Pacientes Ambulatoriais; Saúde Mental 


\section{Colaboradores}

J. G. Almeida participou de todas as etapas de produção do artigo, desde o planejamento até a coleta e análise dos dados, assim como da redação do manuscrito. G. P. Kurita participou da organização e análise dos dados e revisão do artigo. P. E. Braga participou da análise dos dados, interpretação e redação do artigo. C. A. M. Pimenta participou na elaboração do projeto, análise e interpretação dos dados, redação e realizou a revisão crítica deste artigo.

\section{Agradecimentos}

Este trabalho contou com o apoio financeiro da Fundação de Amparo à Pesquisa do Estado de São Paulo (FAPESP) na forma de financiamento (processo $n^{\circ}$. 2008-06046-4). Agradecimentos ao Instituto de Psiquiatria da Faculdade de Medicina da Universidade de São Paulo e ao Estatístico Eduardo Nakano.

\section{Referências}

1. Pimenta CAM, Cruz DALM, Rossetto EG, Dellaroza MSG, Kreling MCGD. Epidemiologia da dor. In: Figueiro JAB, Angelotti G, Pimenta CAM, organizadores. Dor e saúde mental. São Paulo: Editora Atheneu; 2005. p. 3-22.

2. Merskey H, Bogduk N, editors. Classification of chronic pain: descriptions of chronic pain syndromes and definitions of pain terms. Seattle: IASP Press; 1994.

3. Pimenta CAM. Fundamentos teóricos da dor e de sua avaliação. In: Carvalho MMJD, organizador Dor: um estudo multidisciplinar. São Paulo: Summus Editorial; 1999. p. 31-46.

4. Baune BT, Aljeesh Y. Is pain a clinically relevant problem in general adult psychiatry? A clinical epidemiological cross-sectional study in patients with psychiatric disorders. Schmerz 2004; 18:28-37.

5. Kuritzky A, Mazeh D, Lei A. Headache in schizophrenic patients: a controlled study. Cephalalgia 1999; 19:725-7.
6. Chaturvedi S, Michael A. Chronic pain in a psychiatric clinic. J Psychosom Res 1986; 30:347-54.

7. Watson GD, Chandara PC, Merskey H. Relationships between pain and schizophrenia. Br J Psychiatry 1981; 138:33-6.

8. Mahmood T, Romans S, Silverstone T. Prevalence of migraine in bipolar disorder. J Affect Disord 1998; 52:239-41.

9. McIntyre RS, Konarski JZ, Wilkins K, Bouffard B, Soczynska JK, Kennedy SH. The prevalence and impact of migraine headache in bipolar disorder: results from the Canadian Community Health Survey. Headache 2006; 46:973-82.

10. Organização Mundial da Saúde. Classificação de transtornos mentais e de comportamento da CID10: descrições clínicas e diretrizes diagnósticas. 10ạ Revisão. Porto Alegre: Editora Artes Médicas; 1993.

11. Pimenta CAM, Teixeira MJ. Proposta de adaptação do questionário de dor McGill para a língua portuguesa. Rev Esc Enferm USP 1996; 30:473-83. 
12. Wall PD, Melzack R. Textbook of pain. $4^{\text {th }}$ Ed. London: Churchill Livingstone; 1999.

13. Tengan SK. Dor, sintomas depressivos e ansiosos em pré-adolescentes e adolescentes com artrite reumatóide juvenil [Dissertação de Mestrado]. São Paulo: Faculdade de Medicina, Universidade de São Paulo; 2000.

14. Koening TW, Clark, MR Advances in comprehensive pain management. Psychiatr Clin North Am 1996; 19:589-611.

15. Bassit DP, Louzã Neto MR. Complicações decorrentes de úlcera péptica em pacientes com esquizofrenia. Rev ABP-APL 1997; 19:127-32.

16. Fishbain DA. Pain insensitivity in psychosis. Ann Emerg Med 1982; 11:630-2.

17. Smith BH, Elliott AM, Chambers WA, Smith WC Hannaford PC, Penny K. The impact of chronic pain in the community. Fam Pract 2001; 18:292-9.

18. Breivik H, Collett B, Ventafridda V, Cohen R, Gallacher D. Survey of chronic pain in Europe: prevalence, impact on daily life and treatment. Eur J Pain 2006; 10:287-333.

19. Sjogren P, Ekholm O, Peuckmann V, Gronb M. Epidemiology of chronic pain in Denmark: an update. Eur J Pain 2009; 13:287-92.

20. Brattberg G, Thorslund M, Wikman A. The prevalence of pain in a general population. The results of a postal survey in a country of Sweden. Pain 1989; 37:215-22.

21. Neville A, Peleg R, Singer Y, Sherf M, Shvartzman P. Chronic pain: a population-based study. Isr Med Assoc J 2008; 10:676-80.

22. Kreling MCGD, Cruz DALM, Pimenta CAM. Prevalência de dor crônica em adultos. Rev Bras Enferm 2006; 59:509-13.

23. Silva MC, Fassa AG, Valle NCJ. Dor lombar crônica em uma população adulta do Sul do Brasil: prevalência e fatores associados. Cad Saúde Pública 2004; 20:377-85.

24. Dellaroza MSG, Pimenta CAM, Matsuo T. Prevalência e caracterização da dor crônica em idosos não institucionalizados. Cad Saúde Pública 2007; 23:1151-60.
25. Stewart WF, Lipton RB, Celentano DD, Reed ML. Prevalence of migraine headache in the United States. Relation to age, income, race, and other sociodemographic factors. JAMA 1992; 267:64-9.

26. Mathias SD, Kuppermann M, Liberman RF, Lipschutz RC, Steege JF. Chronic pelvic pain: prevalence, health-related quality of life, and economic correlates. Obstet Gynecol 1996; 87:321-7.

27. Bovim G, Schrader H, Sand J. Neck pain in the general population. Spine 1994; 19:1307-9.

28. Ponte C. Lombalgia em cuidados de saúde primários: sua relação com características sociodemográficas. Revista Portuguesa de Clínica Geral 2005, 21:259-67.

29. Almeida ICGB, Sá KN, Silva M, Baptista A, Matos MA, Lessa I. Prevalência de dor lombar crônica na população da cidade de Salvador. Rev Bras Ortop 2008; 43:96-102.

30. Bréder VF, Dantas EHM, Silva MAG, Barbosa LG. Lombalgia e fatores psicossociais em motoristas de ônibus urbano. Fitness \& Performance Journal 2006; 5:294-9.

31. Pahim LS, Menezes AM, Lima R. Prevalência e fatores associados à enxaqueca na população adulta de Pelotas, RS. Rev Saúde Pública 2006; 40:692-8.

32. Spyropoulos P, Papathanasiou G, Georgoudis G, Chronopoulos E, Koutis H, Koumoutsou F. Prevalence of low back pain in greek public office workers. Pain Physician 2007; 10:651-9.

33. Jeste DV, Gladsjo JA, Lindamer LA, Lacro JP. Medical comorbidity in schizophrenia. Schizophr Bull 1996; 22:413-30.

34. Pimenta CAM. Atitudes de doentes com dor crônica frente à dor [Tese de Livre-Docência]. São Paulo: Escola de Enfermagem, Universidade de São Paulo; 1999.

35. Teixeira MJ, Figueiró JAB, Yeng LT, Pimenta CAM Tratamento multidisciplinar do doente com dor In: Carvalho MMJD, organizador. Dor: um estudo multidisciplinar. São Paulo: Summus; 1999. p. 87-139.

Recebido em 06/Mai/2009

Versão final reapresentada em 27/Out/2009

Aprovado em 18/Dez/2009 